\title{
CONNECTION OF TWO CONVENTIONAL ENHANCED TO ENCRYPT COLOR IMAGES
}

\author{
Said Hraoui \\ LIASSE, ENSA, SMBA University \\ Fez, Morocco \\ said.hraoui@usmba.ac.ma,
}

\author{
Abdellatif JarJar \\ Moulay Rachid High School, \\ Taza, Morocco \\ abdoujjar@gmail.com
}

\begin{abstract}
This document introduces a new cryptosystem mixing two improvement standards generally used for text encryption, in order to give birth a new color image encryption algorithm capable of dealing with known attacks. Firstly, two substitution matrixes attached to a strong replacement function will be generated for advanced Vigenere technique application. At the end of this first round, the output vector is subdivided into size blocks according to the used chaotic map, for acting a single enhanced Hill circuit insured by a large inversible matrix. A detailed description of such a large involutive matrix constructed using Kronecker products will be given. accompanied by a dynamic translation vector to eliminate any linearity. A solid chaining is established between the encrypted block and the next clear block to avoid any differential attack. Simulations carried out on a large volume of images of different sizes and formats ensure that our approach is not exposed to any known attacks.
\end{abstract}

\section{Article Highlights}

This new algorithm is the mixture result of two deeply improved classical systems which we mention the most important changes made.

$>$ New size substitution matrix $(256,256)$ Construction

$>$ Strong replacement functions definition

> Improved Vigenere technology application

> Kronecker products Theoretical reminder

$>$ Kronecker product Application

$>$ Large invertible matrix design

$>$ Single enhanced Hill revolution application

\section{Notation}

$$
\left\{\begin{array}{c}
G_{t}=Z / t Z \text { ring } \\
A(j:): \text { Line number } j \text { of matrix } A \\
A(: j): \text { colunm number } j \text { of matrix } A \\
\oplus: \text { Xor operator } \otimes: \text { Kronecker product } \\
E(x): \text { The whole part of the real number } x
\end{array}\right.
$$

Key word: Vigenere grid; Involutive matrix; Chaotic map; Broadcast function; genetic operator 


\section{INTRODUCTION}

The rapid development of chaos theory in mathematics provides researchers with opportunities to further improve some classic encryption systems. In front of this great security focus, many techniques for color image encryption have flooded the digital world, mostly exploiting number theory and chaos [1,2]. Others are attempting to update their policies by improving some classical techniques, such as Hill [3-4], Cesar, Vignere [5 - 6], Feistel [7 - 8].

\section{1) Vigenere's classical technique}

This technology is based on static $(V)$ matrix defined by the following algorithm

$$
\text { algorithm1 }\left\{\begin{array}{c}
\text { Fist Row } \\
\text { For } i=1 \text { to } 26 \\
V(1, i)=i \\
\text { Next } i \\
\text { folloying } R \text { Rows } \\
\text { For } i=2 \text { to } 26 \\
\text { For } j=1 \text { to } 26 \\
V(i, j)=V(i-1,(j+1), 26) \\
\text { Next } j, i
\end{array}\right.
$$

Let $(P)$ : plain text, $(C)$ : cypher text; $(K)$ : Encryption key, $(V)$ Vigenere matrix and $(l)$ : length of clear text. So

$$
\text { equation } 1\left\{\begin{array}{cc}
C_{i}=V\left(P_{i}, K_{i}\right)=\left(P_{i}+K_{i}\right) & \bmod 26 \\
P_{i}=V\left(C_{i}, K_{i}\right)=\left(P_{i}-K_{i}\right) & \bmod 26
\end{array}\right.
$$

Even though Vigenere's matrix was known, the encryption was able to withstand several centuries. But, Babagh's cryptanalysis is not efficient in not knowing the size of the encryption key. Several attempts to improve Vigenere's technique have invaded the digital world we quote $[9-10]$. In this work, the new structure of the substitution matrix and its attached replacement function will be described in detail.

\section{2) Hill's classical technique}

Hill's method is a classic symmetric encryption system that uses a linear transformation provided by an invertible matrix in a carefully selected ring [11 - 12].

$(C)$ :plain text $\left(C^{\prime}\right)$ : cypher text; $(K)$ : Encryption key, the method is defined by the following linear formula

$$
\text { Equation } 2\left\{\begin{array}{c}
C^{\prime}=K C \\
S o \\
C=K^{-1} C^{\prime}
\end{array}\right.
$$

Unfortunately, this technique is still exposed to known attacks. Since then, over time, several improvements have been made to the system [13-14]. The improvement of the classic Hill technique tracked in this document is to prove the construction of a large invertible matrix whose size will be randomly calculated based on the chaotic graph used. Some advanced techniques in the same axis should be taken into consideration $[15-16]$. 


\section{3) Our contribution}

Our contribution in this article is to couple two conventional deeply improved systems to better adapt to color image encryption. These improvements, made on the two classic systems are

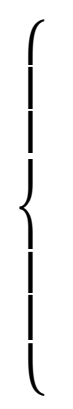

$$
\text { Vigenere }
$$

New substitution matrices construction

New attached replacement functions definition

Application of a diffusion ensured by two size maatrix $(256,256)$

Hill

Large key matrix Construction

Applying an affine transformation

Dynamic translation vector

\section{THE PROPOSED METHOD}

Based on chaos, our new technology is divided into three main stages

$$
\left\{\begin{array}{c}
\text { first phase: } \\
\text { action on a single pixel with broadcast installation } \\
\text { Second phase: } \\
\text { subdivision of the image into blocks of size }(4 r) \\
\text { Third phase } \\
\text { Construction of an invertible matrix of size }(4 r, 4 r) \\
\text { action on blocks of }(4 r) \text { pixels by a Hill lap }
\end{array}\right.
$$

The image below shows this technology steps 


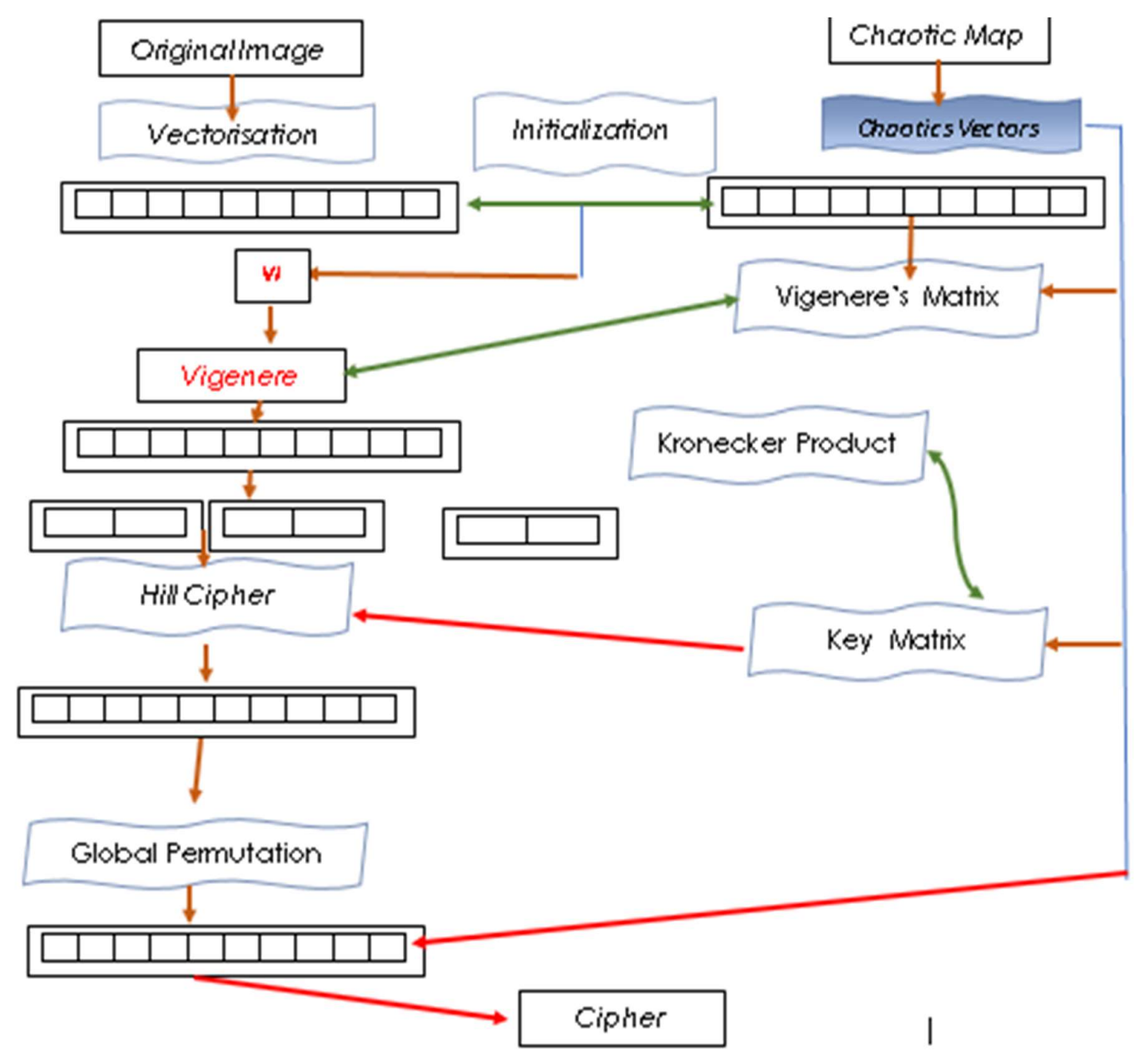

Figure2: Encryption scheme

This new cryptosystem is based on the following axes AXE 1: CHAOTIC SEQUENCES DEVELOPMENT

In order to build a new algorithm using a single-encryption key, we will use the $2 \mathrm{~d}$ logistics map. This choice is due to the simplicity of its development and its high sensitivity to the initial parameters.

\section{1) 2 D Logistics Map}

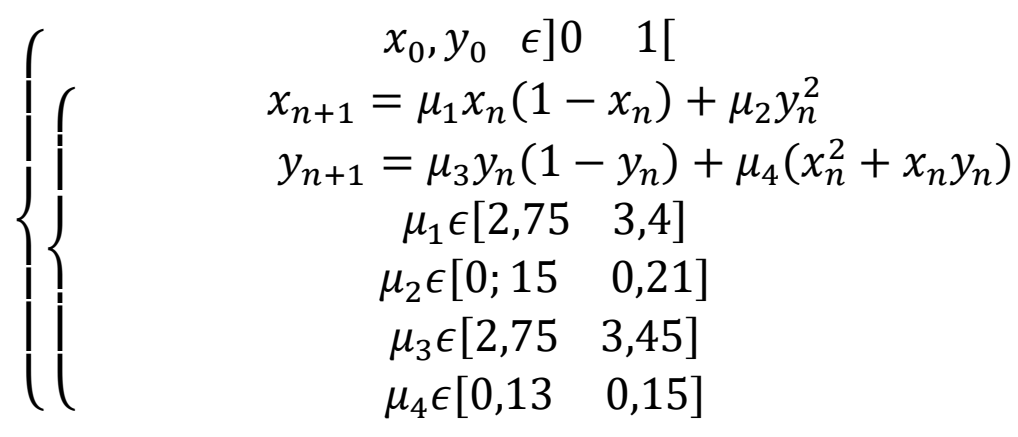




\section{2) Chaotic used vector design}

Our work requires the construction of three chaotic vectors $(C L),(K R)$ and $(K L)$, with a coefficient in $\left(G_{256}\right)$, and two $(C R),(C V)$ binary vector will be regarded as the control vector. This construct is seen by the following algorithm

$$
\text { Algorithm }\left\{\begin{array}{c}
\text { for } i=1 \text { to } 3 \mathrm{~nm} \\
C L(i)=\bmod \left(E\left(\frac{x(i)+\sup (x(i), y(i))}{2} * 10^{11}, 254\right)+1\right) \\
K L(i)=\bmod \left(E\left(\frac{x(i) * y(i)+\inf (x(i), y(i)) v(i)}{2} * 10^{10}, 253\right)+2\right) \\
K R(i)=E\left(\frac{K L(i)+C L(i)}{2}\right) \\
\text { if } x(i) \geq y(i) \text { then } \\
C R(i)=0 \text { else } C R(i)=1 \\
\text { end if } \\
\text { if } C l(i)<K L(i) \text { then } \\
C V(i)=1 \\
\text { else } C V(i)=0 \\
\text { end if } \\
\text { Next } i
\end{array}\right.
$$

\section{3) Global permutation Design}

Sorting the $(\mathrm{CR})$ vector in a large decreasing order generates the $(\mathrm{PH})$ global permutation This method is illustrated by the following algorithm

$$
\text { Algorithm3 }\left\{\begin{array} { c } 
{ k = 1 } \\
{ \text { For } i = 3 \text { nm to } 1 } \\
{ \text { If } C R ( i ) = 0 \text { Then } P H ( i ) = k } \\
{ k = k + 1 } \\
{ \text { end if } } \\
{ \text { Next } i }
\end{array} \quad \left\{\begin{array}{c}
\text { For } i=1 \text { to } 3 \mathrm{~nm} \\
\text { If CR }(i)=0 \text { Then } P H(i)=k \\
k=k+1 \\
\text { end if } \\
\text { Next } i
\end{array}\right.\right.
$$

Example:

\begin{tabular}{|l|l|l|l|l|l|l|l|l|l|l|l|l|l|l|l|l|l|l|l|l|}
\hline (CR) & 0 & 0 & 1 & 1 & 1 & 0 & 1 & 0 & 1 & 1 & 0 & 1 & 0 & 1 & 1 & 0 & 0 & 1 & 1 & 0 \\
\hline
\end{tabular}

\begin{tabular}{|l|l|l|l|l|l|l|l|l|l|l|l|l|l|l|l|l|l|l|l|l|}
\hline Sort & 9 & 8 & 10 & 11 & 12 & 7 & 13 & 6 & 14 & 15 & 5 & 16 & 4 & 17 & 18 & 3 & 2 & 19 & 20 & 1 \\
\hline
\end{tabular}

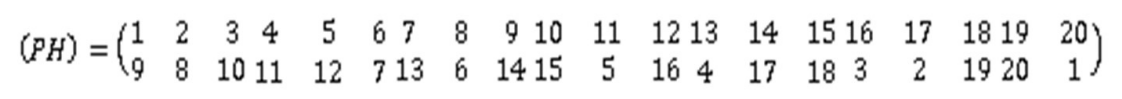

\section{INSTALL THE NEW ENCR YPTION METHOD}

Based on chaos, our new technology is based on two encryption processes each using a deep improvement of a classic system. 


\section{AXE 2: VIGENERE UPGRADE}

In the first stage, Vigenere's technology was greatly modified by integrating the new substitution matrix provided by the new powerful replacement function.

\section{1) Original image Vectorization}

After the three $(R G B)$ color channels extraction and their conversion into size vectors $(\boldsymbol{V r}),(\boldsymbol{V} \boldsymbol{g}),(\boldsymbol{V} \boldsymbol{b})(\mathbf{1}, \boldsymbol{n m})$ each, a concatenation is established to generate a vector $\boldsymbol{X}\left(\boldsymbol{x}_{1}, \boldsymbol{x}_{2}, \ldots \ldots, \boldsymbol{x}_{\mathbf{3 n m}}\right)$ of size $(1,3 \mathrm{~nm})$. This operation is described by the following algorithm

$$
\text { Algorithm } 5\left\{\begin{array}{c}
\text { for } i=2 \text { tonm } \\
X(3 i-2)=V r(i) \\
X(3 i-1)=V g(i) \\
X(3 i)=V b(i) \\
\text { Next } i
\end{array}\right.
$$

\section{2) Initialization Value Design}

The(IV1) initialization value must be recalculated to change the value of the starting pixel. Ultimately, the (IV1) value is provided by the next algorithm

$$
\text { Algorithm } 6\left\{\begin{array}{c}
\text { for } i=2 \text { to } l \\
\text { if } C V(i)=0 \text { then } \\
v_{1}=v_{1} \oplus X(i) \oplus C L(i) \\
\text { Else } \\
v_{1}=v_{1} \oplus X(i) \oplus K L(i) \\
\text { Next } i
\end{array}\right.
$$

This initialization value is set only to modify the value of the seed pixel and start the encryption process. By implementing chaotic mapping in the calculation of the initialization value, the problem of uniform image color (black, white ,... ) can be solved.

\section{3) Vigenere's advanced methods}

This new technology requires the establishment of two $(V G)$ and $(V D)$ substitution matrices through the process described by the following steps

$>$ permutation $(R P)$ obtained by descending ordering the first 256 values of the sequence $(U)$

$>$ permutation $(R R)$ obtained by increasing the ordering the first 256 values of the sequence $(V)$,

with the following restrictions

$$
\text { Equation } 7 \quad\left\{\begin{array}{l}
\text { if } R P(i)=256 \text { the } R P(i)=0 \\
\text { if } R R(i)=256 \text { the } R R(i)=0
\end{array}\right.
$$

This new construction is entirely supervised by the vector $(C R)$ seen as a decision vector. It is given by the following algorithm 


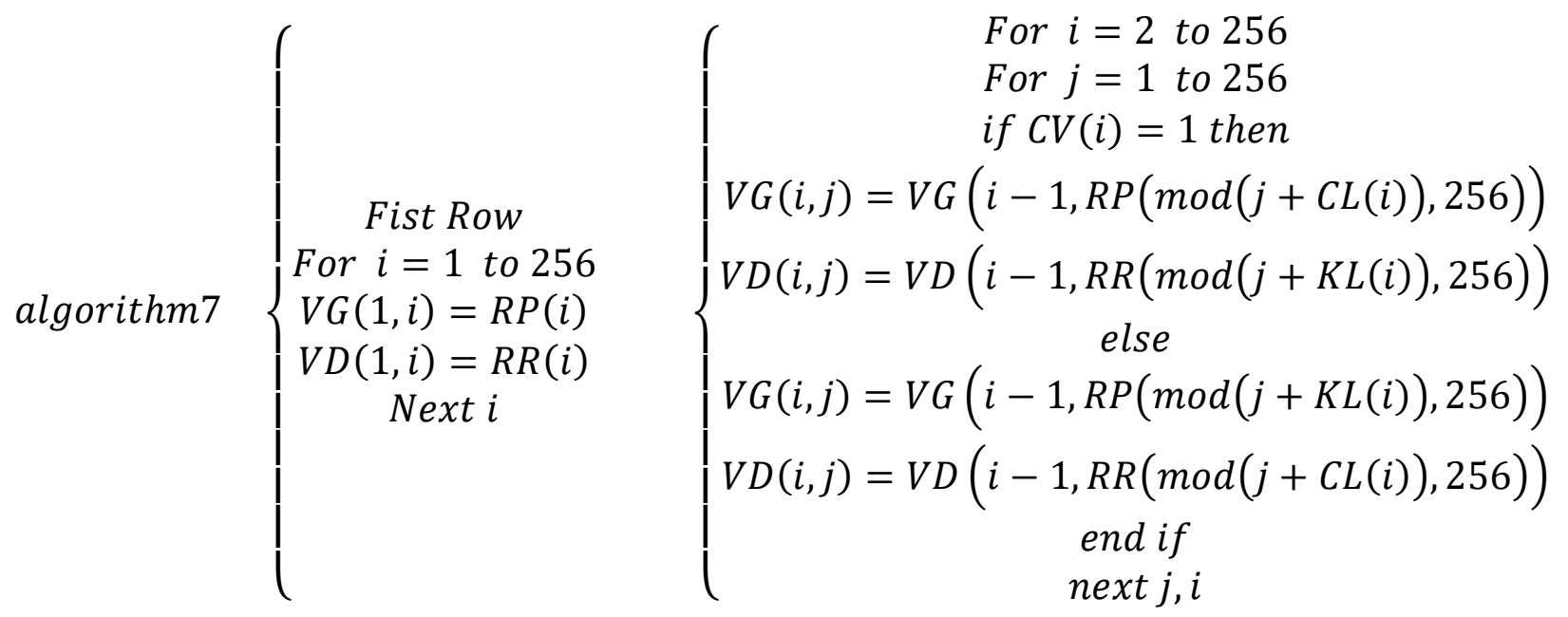

We note that the construction of the two matrices is completely determined by the $(\mathrm{CV})$ decision vector

Example: in $\left(\boldsymbol{G}_{\mathbf{8}}\right)$

\begin{tabular}{|c|c|c|c|c|c|c|c|c|c|c|c|c|}
\hline (VG) & 1 & 2 & 3 & 4 & 5 & 6 & 7 & & 0 & $C R$ & $\mathrm{Kl}$ & $\mathrm{dl}$ \\
\hline 1 & 3 & 5 & 0 & 6 & 2 & 7 & 1 & & 4 & & & \\
\hline 2 & 2 & 7 & 1 & 4 & 3 & 5 & 0 & & 6 & 1 & 5 & 4 \\
\hline 3 & 4 & 3 & 5 & 0 & 6 & 2 & 7 & & 1 & 1 & 3 & 5 \\
\hline 4 & 2 & 7 & 1 & 4 & 3 & 5 & 0 & & 6 & 0 & 3 & 4 \\
\hline 5 & 0 & & 2 & 7 & 1 & 4 & 3 & & 5 & 1 & 4 & 2 \\
\hline
\end{tabular}

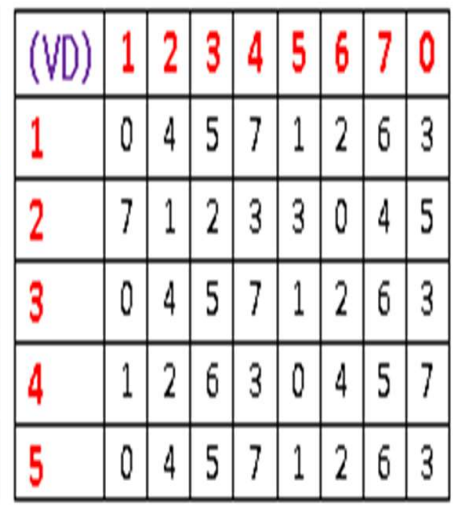

a) New Vigenere's mathematical expression

The classical Vigenere transformation expression is given by the following formula Equation8 $\{\quad Y(i)=V G(C L(i), X(i))$

The following equation illustrates the effective expression of the $Y(i)$ image of the pixel $X(i)$ through the new Hill technology.

$$
\text { Equation9 }\left\{\begin{array}{c}
\text { if } C R(i)=0 \text { then } \\
Y(i)=V D(K L(i), V G(K R(i),(\bmod (c * X(i), 256) \oplus C L(i))) \\
\text { With;a;c } \in G_{256}^{*} \\
\text { Let }\left\{\begin{array}{c}
a=\bmod \left(2 * \bmod \left(\sum_{i=1}^{n} C L(i)\right)+1, \quad 256\right) \\
c=\bmod \left(2 * \bmod \left(\sum_{i=1}^{n m} K L(i)\right)+1,256\right)
\end{array}\right)
\end{array}\right.
$$


We noticed that this new expression is firmly attached to the $(C R)$ decision vector, and uses two general substitution matrices to contain powerful substitution functions.

\section{2) First Encryption Process}

With a powerful broadcast function, this first encryption process will protect the system from differential attacks and increase the time complexity of the attack. The figure below illustrates the first encryption process improved using only Vigenere

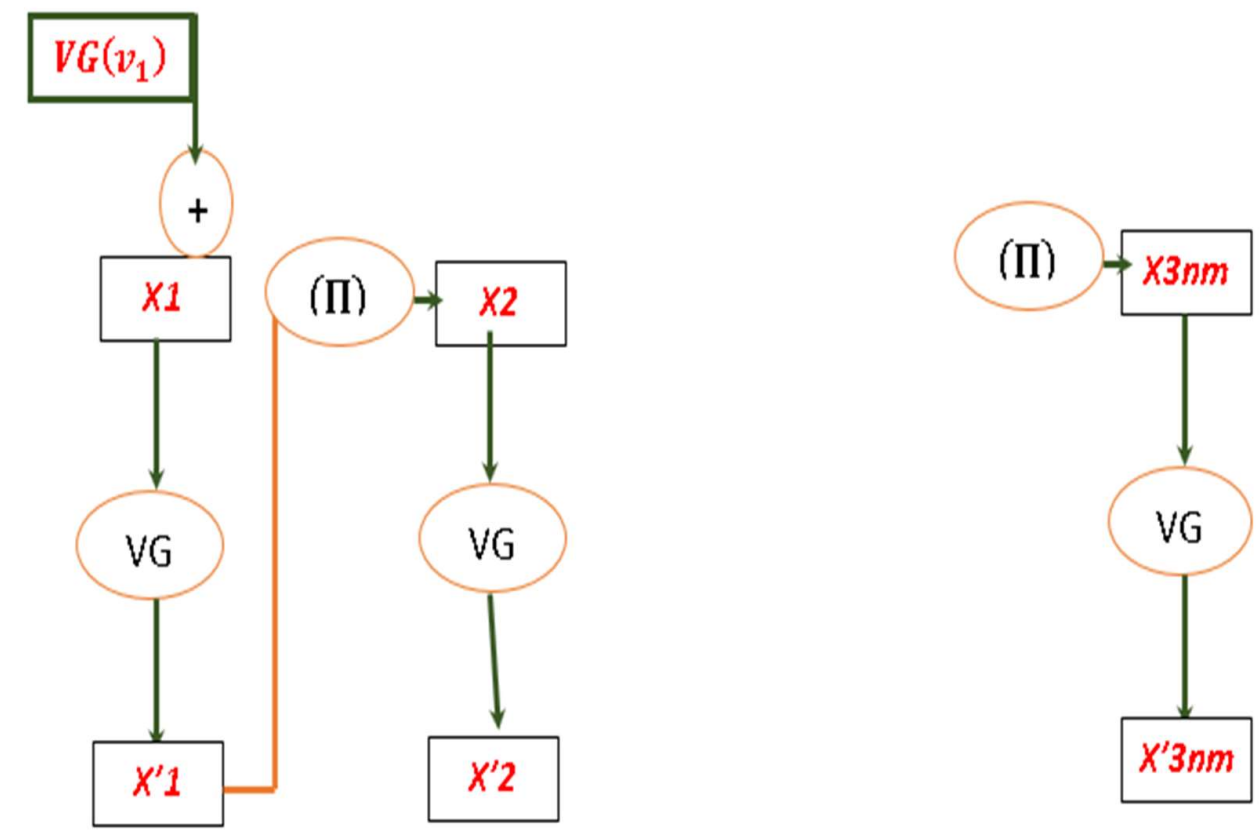

Figure4 : Vigenere Encryption

$\left\{\begin{array}{l}\text { (VG): advanced substitution matrix } \\ \text { (П): New diffusion function }\end{array}\right.$

(I) The new diffusion function using matrix $(V D)$ is described by the following formula Equation $10 \quad\left\{\quad \Pi(X(i+1))=V D\left(K R(i), X^{\prime}(i) \oplus X(i+1)\right)\right.$

This schema is translated by the algorithm below

$$
\text { Algorithm8 }\left\{\begin{array}{c}
X^{\prime}(1)=V G\left(K R(1), V D\left(C L(1), v_{1}\right) \oplus X(1)\right) \\
\quad F o r i=2 \text { to } 3 \mathrm{~nm} \\
\Pi(X(i))=V D\left(K R(i), X^{\prime}(i-1) \oplus X(i)\right) \\
\text { if } C R(i)=0 \text { then }
\end{array}\right)
$$

We note that this first step uses only substitutions, which ensures an extreme speed in the execution. The output vector $\boldsymbol{X}^{\prime}\left(\boldsymbol{x}_{1}^{\prime}, \boldsymbol{x}_{2}^{\prime}, \ldots \ldots, \boldsymbol{x}_{3 \mathbf{n} \boldsymbol{m}}^{\prime}\right)$, will undergo a second encryption attempt. 


\section{AXE 3: APPL YING HILL UPGRADE}

Before starting the second encryption stage, we will introduce in detail some mathematical knowledge useful for constructing inversible matrix and, install a hill lap.

\section{1) Math reminder}

Several new knowledges with mathematical bases must be clarified

\section{a. related information}

In this section, we will introduce the concept of diastereomeric matrices and Kronecker products used to construct large invertible matrices.

\section{i. Involutive matrix}

\section{a) Definition}

$A$ is an involutive matrix if and only if we have

$$
\text { Equation } 14 \quad A^{-1}=A
$$

In other words

$$
\text { Equatiopn15 } \quad A^{2}=I \text { I identity matrix }
$$

\section{b. Building the $(A)$ matrix}

Assume that the size of $(\boldsymbol{A})$ matrix is $\left(2 r_{h}, 2 r_{h}\right)$, and is defined by block as follows

We got

$$
A=\left(\begin{array}{ll}
A_{1} & A_{2} \\
A_{3} & A_{4}
\end{array}\right) A_{i} \text { of size }\left(r_{h}, r_{h}\right)
$$

$A^{2}=\left(\begin{array}{ll}A_{1} & A_{2} \\ A_{3} & A_{4}\end{array}\right)\left(\begin{array}{ll}A_{1} & A_{2} \\ A_{3} & A_{4}\end{array}\right)=\left(\begin{array}{cc}A_{1}^{2}+A_{3} A_{2} & A_{2} A_{1}+A_{4} A_{2} \\ A_{1} A_{3+} A_{3} A_{4} & A_{4}^{2}+A_{2} A_{3}\end{array}\right)=\left(\begin{array}{ll}I & 0 \\ 0 & I\end{array}\right) \quad$ I: Identity Matrix

Since matrix $(A)$ is involutive, we get

$$
\text { Equation16 }\left\{\begin{array}{l}
A_{1}^{2}+A_{2} A_{3}=I \\
A_{2} A_{1}+A_{4} A_{2}=0 \text { with } 0: \text { Null matrix } \\
A_{1} A_{3+} A_{3} A_{4}=0 \\
A_{4}^{2}+A_{3} A_{2}=I
\end{array}\right.
$$

So

$$
\text { Equation17 }\left\{\begin{array}{l}
A_{2} A_{3}=I-A_{1}^{2}=\left(I-A_{1}\right)\left(I+A_{1}\right) \\
A_{3} A_{2}=I-A_{4}^{2}=\left(I-A_{4}\right)\left(I+A_{4}\right)
\end{array}\right.
$$

Since $\left(A_{1}\right)$ matrix is given randomly, other matrices can be selected by the following formula

$$
\text { Equation } 18\left\{\begin{array} { c } 
{ A _ { 2 } = k ( I - A _ { 1 } ) } \\
{ A _ { 3 } = k ^ { - 1 } ( I + A _ { 1 } ) ( k \in G _ { 2 5 6 } ^ { * } ) } \\
{ A _ { 4 } = 2 5 6 - A _ { 1 } }
\end{array} \text { and } \left\{\begin{array}{l}
A_{1} \neq I \\
A_{1} \neq 0
\end{array}\right.\right.
$$


Or We can take

Equation19 $\left\{\begin{array}{c}A_{2}=k\left(I+A_{1}\right) \\ A_{3}=k^{-1}\left(I-A_{1}\right) \\ A_{4}=256-A_{1}\end{array}\right.$ This gives $A=\left(\begin{array}{cc}A_{1} & k\left(I-A_{1}\right) \\ k^{-1}\left(I+A_{1}\right) & 256-A_{1}\end{array}\right)$

\section{Example}

$$
\text { In } \boldsymbol{G}_{\mathbf{8}} \quad A_{1}\left(\begin{array}{ll}
1 & 3 \\
2 & 5
\end{array}\right) \quad k=3 \text { and } k^{-1}=3 \text { So } \quad A=\left(\begin{array}{llll}
1 & 3 & 0 & 7 \\
2 & 5 & 2 & 4 \\
6 & 1 & 7 & 5 \\
6 & 2 & 6 & 3
\end{array}\right)
$$

Under these conditions, the obtained matrix $(A)$ is involute. This choice is not unique, and it increases the complexity of matrix $(A)$ reconstruction.

\section{2) Kronecker matrix product}

Matrix theory is widely used in cryptography. Due to its difficulties, classical computing techniques are rarely used. For example, it is difficult to reverse the large matrix using conventional methods. At present, the Kronecker product and the tensor product are not only connected with physics or biology, but also take a safe path in cryptography. We are going to take advantage of the Kronecker product to increase the size of the involutive matrix.

\section{a. Definition}

Let $A=\left(a_{i, j}\right)$ of size $(n, m)$ and Let $B=\left(b_{i, j}\right)$ of size $(p, q)$, We call the Kronecker product of $A$ and $B$, denoted by

$$
\text { Equation } 20 \quad C=A \otimes B=\left(\begin{array}{ccc}
a_{1,1} B & \cdots & a_{1, m} B \\
\vdots & \ddots & \vdots \\
a_{n, 1} B & \cdots & a_{n, m} B
\end{array}\right) \text { of size }(n p ; m q)
$$

\section{Example}

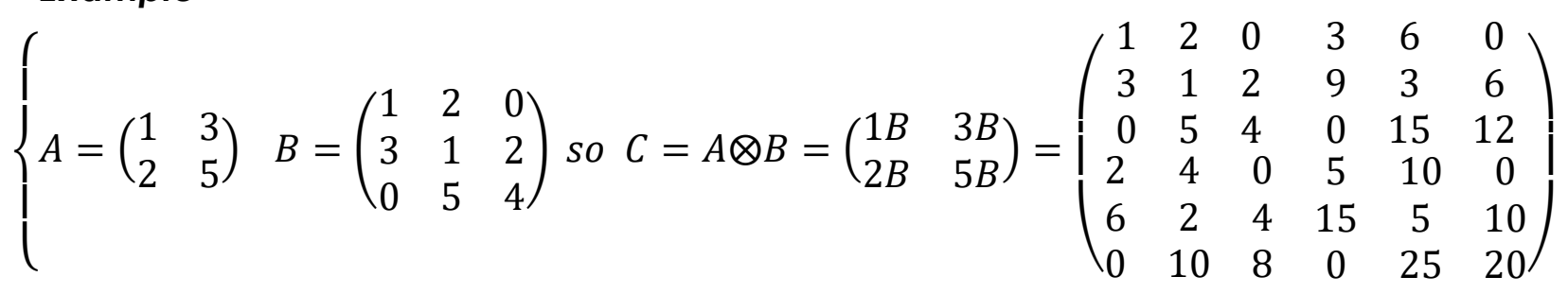

\section{b. Kronecker Product Properties}

The product is not commutative

$$
A \otimes B \approx B \otimes A
$$

\section{c. Reversibility}


Note:

Equation $21\left\{\begin{array}{c}\text { if } A \text { is invertible and if } B \text { is invertible Then } \\ (A \otimes B)^{-1}=A^{-1} \otimes B^{-1}\end{array}\right.$

If $A$ and $B$ are involutive matrices, then their Kronecker product is involutive and we have

\section{3) ( $\left.X^{\prime}\right)$ Vector Adaptation}

$$
\text { Equation } 22(A \otimes B)^{-1}=A^{-1} \otimes B^{-1}=A \otimes B
$$

In order to facilitate the implementation of the second encryption stage, the vector $\left(X^{\prime}\right)$ must be cut into blocks uniform, we are going to calculate two constants $r_{1}$ and $r_{2}$

We put

$$
\text { Equation } 23\left\{\begin{array}{l}
\boldsymbol{r}_{\mathbf{1}}=\left(\bmod \left(\frac{1}{n} \sum_{i=1}^{n}|C L(i)-K L(i)|, 4\right)+2\right) \text { so } 2 \leq \boldsymbol{r}_{\mathbf{1}} \leq \mathbf{5} \\
\boldsymbol{r}_{\mathbf{2}}=\left(\bmod \left(\frac{1}{m} \sum_{i=1}^{m}|C L(i)-K R(i)|, 3\right)+2\right) \text { so } 2 \leq \boldsymbol{r}_{\mathbf{2}} \leq \mathbf{4}
\end{array}\right.
$$

$$
16 \leq r=4 \boldsymbol{r}_{\mathbf{1}} \boldsymbol{r}_{\mathbf{2}} \leq \mathbf{8 0}
$$

Therefore, the new vector size $\left(X^{\prime}\right)$ can be obtained by the following formula

$$
\text { Equation } 24 \quad\left\{\begin{array}{c}
\text { let } 3 \mathrm{~nm} \equiv s[\boldsymbol{r}] \\
l=3 \mathrm{~nm}-\mathrm{s} \\
t=\frac{l}{r}
\end{array}\right.
$$

By truncating the last $(s)\left(X^{\prime}\right)$ pixel, and placing it in the $(X D)$ vector after modification, the (XT) adaptive vectorcan be obtained by the following algorithm

$$
\text { Algorithm } 9\left\{\begin{array}{c}
\text { for } i=1 \text { to } l \\
X T(i)=X^{\prime}(i) \\
\text { Next } i \\
\text { Amputated pixel storage } \\
\text { for } i=1 \text { to } s \\
\text { if } C R(i+l)=0 \text { then } \\
X D(i)=X^{\prime}(i+l) \oplus C L(i+l) \\
\text { else } \\
X D(i)=0 \text { then }(i+l) \oplus K L(i+l) \\
\text { end if } \\
\text { Next } i \\
\text { end if }
\end{array}\right.
$$

\section{4) Switching to a size matrix}

The following figure illustrates the transaction from vector to size matrix $(t, r)$ used in the Hill circuit 


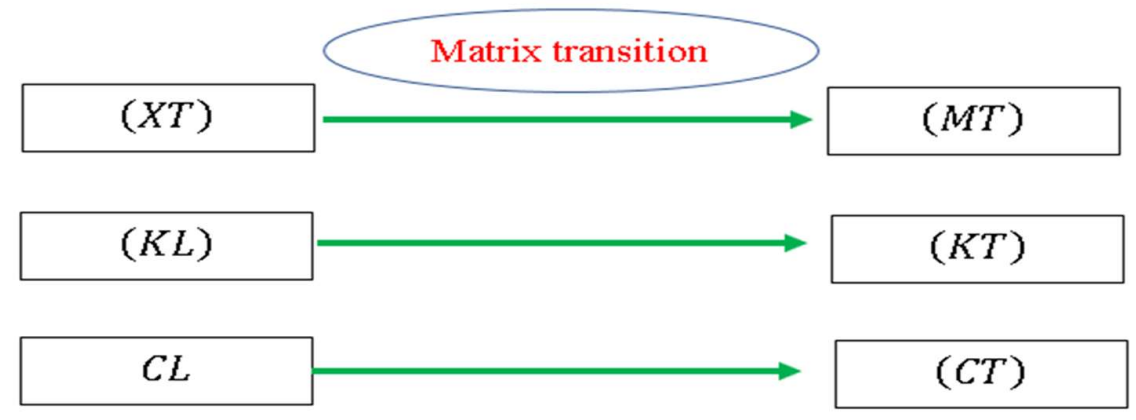

Figure6: Transition to matrices $(\boldsymbol{t}, \boldsymbol{r})$

Hill's classical method is predominantly oriented on invertible matrices in accurately indexed rings. Inverting a large matrix remains the main challenge of this technique, which prompts scholars to use only small matrices typically of size $<=4$.

\section{2) New Hill matrix Development}

In our algorithm, in order to overcome this problem, we will introduce in detail a new simple method of constructing large invertible matrix based on involute matrix.

Let $\left(A_{1}\right)$ be an arbitrary matrix of size $\left(r_{1}, r_{1}\right)$ and let the matrix $(A)$ defined by

$$
\text { Equation } 25 \quad A=\left(\begin{array}{cc}
A_{1} & k\left(I-A_{1}\right) \\
k^{-1}\left(I+A_{1}\right) & 256-A_{1}
\end{array}\right)\left(k \in G_{256}^{*}\right) \text { of size }\left(2 r_{1}, 2 r_{1}\right)
$$

Let $\left(B_{1}\right)$ be an arbitrary matrix of size $\left(r_{2}, r_{2}\right)$ and let the matrix $(B)$ defined by

$$
\text { Equation } 26 B=\left(\begin{array}{cc}
B_{1} & h\left(I-B_{1}\right) \\
h^{-1}\left(I+B_{1}\right) & 256-B_{1}
\end{array}\right)\left(h \in G_{256}^{*}\right) \text { of size }\left(2 r_{2}, 2 r_{2}\right)
$$

The two matrices $(A)$ and $(B)$ are involutive

We put

$$
\text { Equation } 27 H=A \otimes B
$$

The matrix $(C)$ is invertible and involutive of size $(4 r, 4 r)$. The matrix $(C)$ is condadate for a key Hill matrix.

\section{3) New encryption matrix construction}

This second encryption process is illustrated by the following figure 

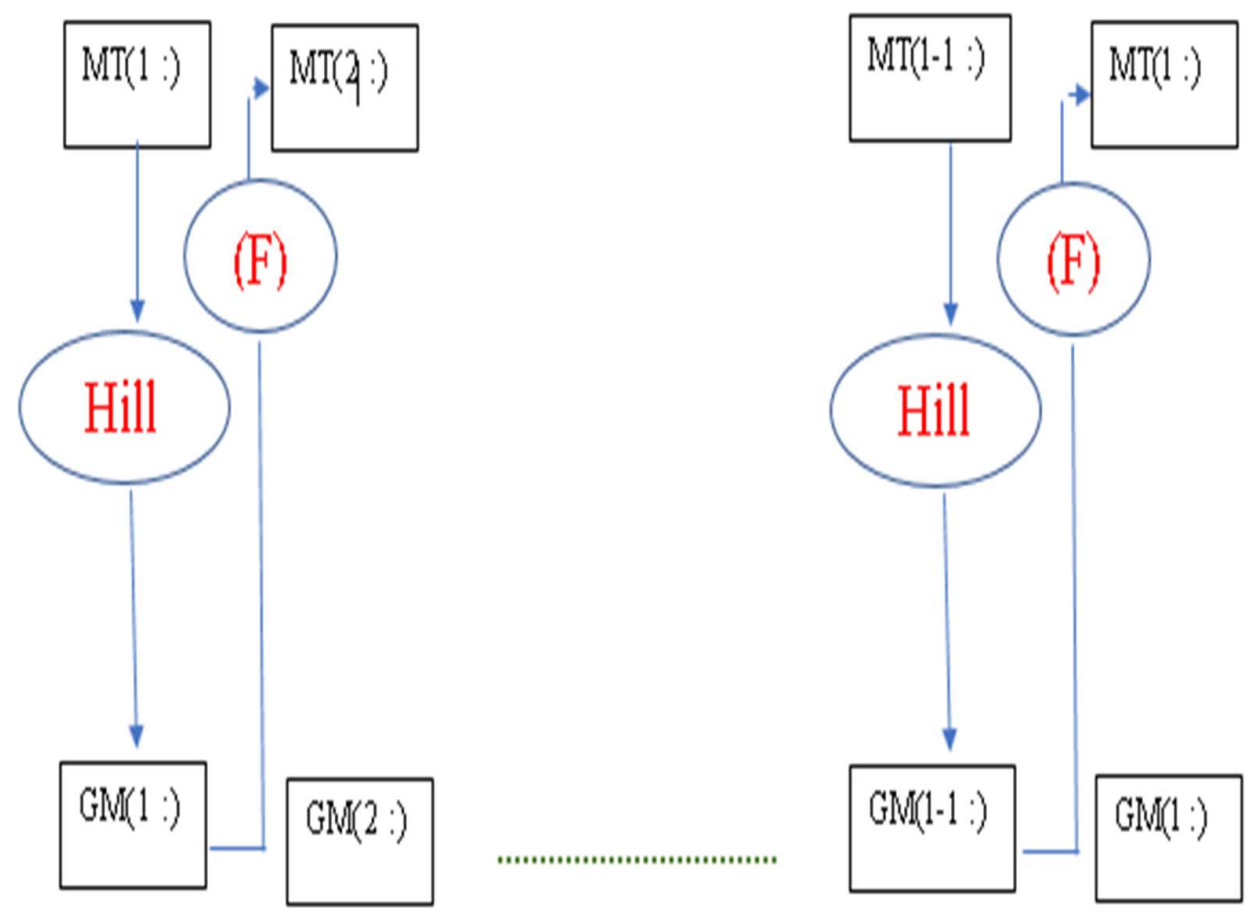

Figure6: Hill Encryption

$\left\{\begin{array}{c}(H I L L): \text { enhanced HILL function } \\ (F): \text { New difusion function }\end{array}\right.$

\section{4) Enhanced HILL function expression}

$$
\text { Algorithm11 Hill }\left\{\begin{array}{c}
\text { if } C V(k)=0 \text { then } \\
G M(k:)=H * M T(k:) \oplus K T(k:) \\
\text { else } \\
G M(k:)=H * M T(k:) \oplus C T(k:)
\end{array}\right.
$$

Build diffusion to increase the impact of the avalanche effect

$$
\text { Equation } 28\{F(M T(k+1:))=G M(k:) \oplus M T(k+1:) \oplus C T(k:)
$$

An insertion of the pixels of the vector $(X D)$ at the end of the obtained vector $(C X)$ on which the permutation $(\mathrm{PH})$ is established by the following algorithm

$$
\text { Algorithm } 16\left\{\begin{array}{c}
\text { for } i 1=1 \text { to } 3 \mathrm{~nm} \\
X C(i)=C X(P H(i)) \\
\text { Next } i
\end{array}\right.
$$

The vector $(X C)$ represents the encrypted image. 


\section{STEP 5: DECR YPTION ENCR YPTED IMAGES}

The solid chaining establishes between the encrypted block and the next clear block in the broadcast process, forcing us to start decryption from the last block using the opposite functions. So, the decryption process should follow these steps

* Read the encrypted image and switch to vector $(X C)$

- Application of the permutation (HP) inverse of the permutation $(P H)$

$$
\begin{gathered}
\text { Algorithm } 17\left\{\begin{array}{c}
\text { For } i=1 \text { to } 3 \mathrm{~nm} \\
H P(P H(i))=i \\
\text { Next } i
\end{array}\right. \\
\text { Algorithm } 18\left\{\begin{array}{c}
\text { For } i=1 \text { to } 3 \mathrm{~nm} \\
C X(i)=X C(H P(i) \\
\text { Next } i
\end{array}\right.
\end{gathered}
$$

* Recalculates the value $(\boldsymbol{r})$ size of the subdivision blocks

* Recalculates $(A)$ and $(B)$

* Reverse Hill application $\left(A^{-1}=A, B^{-1}=B\right) H=A \otimes B=H^{-1}$

\section{1) Mathematical HILL inverse expression}

$$
\text { Algorithm11 } \operatorname{HILL}^{-1}\left\{\begin{array}{c}
\text { if } C V(k)=0 \text { then } \\
M T(k:)=H^{-1}(M T(k:) \oplus K T(k:)) \\
\text { else } \\
M T(k:)=H^{-1}(M T(k:) \oplus C T(k:))
\end{array}\right.
$$

- Reconstruct the image vector and add vector pixels (XD) to the queve

* Reverse Vigenere application

\begin{tabular}{|c|c|c|c|c|c|c|c|c|c|c|c|c|c|c|c|c|c|c|c|}
\hline (VG) & 1 & 2 & 3 & 4 & 5 & 57 & 0 & $C R$ & $K L$ & $C L$ & (GV) & 1 & 2 & 3 & 4 & 5 & 6 & 7 & 0 \\
\hline 1 & 3 & 5 & 0 & 6 & 2 & 71 & 14 & & & & 1 & 7 & 5 & 1 & 0 & 2 & 4 & 6 & 3 \\
\hline 2 & 2 & 7 & 1 & 4 & 3. & 50 & $\begin{array}{lll}0 & 6 \\
\end{array}$ & 1 & 5 & 4 & 2 & 3 & 1 & 5 & 4 & 6 & 0 & 2 & 7 \\
\hline 3 & 4 & 3 & 5 & 0 & 6 & 27 & 7 & 1 & 3 & 5 & 3 & 0 & 6 & 2 & 1 & 3 & 5 & 7 & 4 \\
\hline 4 & 2 & 7 & 1 & 4 & 3 & 5 & 06 & 0 & 3 & 4 & 4 & 3 & 1 & 5 & 4 & 6 & 0 & 2 & 7 \\
\hline 5 & 0 & & 2 & 7 & 1 & 47 & 5 & 1 & 4 & 2 & 5 & 5 & 3 & 7 & 6 & 0 & 2 & 4 & 1 \\
\hline
\end{tabular}

$$
\text { Algorithm } 20\left\{\begin{array}{c}
\text { for } i=1 \text { to } 256 \\
\text { for } j=1 \text { to } 256 \\
G V(i, V G(i, j))=j \\
D V((i, V D(i, j))=j \\
\text { Next } j, i
\end{array}\right.
$$

\section{Example}

By following the same logic of Vigenere's traditional technique, we obtain

$$
\text { Equation } 21 \quad\left\{\begin{array}{c}
\text { if } z=V G(y, x) \\
\text { Then } \\
x=G V(y, z)
\end{array}\right.
$$




\section{2) Mathematical Vigenere inverse expression}

We have

So

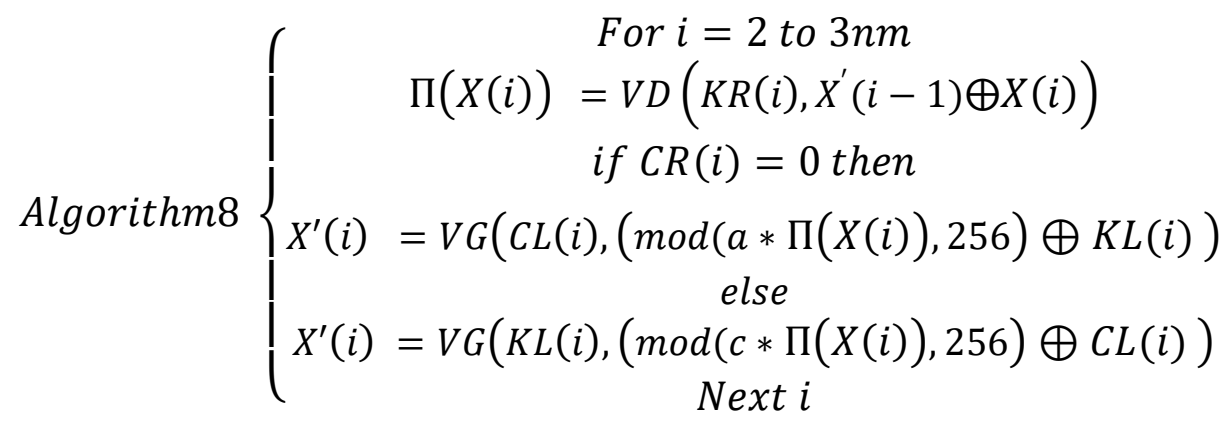

$$
\left\{\begin{array}{c}
X^{\prime}(i)=V G(C L(i),(\bmod (a * \Pi(X(i)), 256) \oplus K L(i)) \\
\left(\bmod (a * \Pi(X(i)), 256) \oplus K L(i)=G V\left(C L(i), X^{\prime}(i)\right) .\right. \\
\left(\bmod (a * \Pi(X(i)), 256)=G V\left(C L(i), X^{\prime}(i)\right) \oplus K L(i)\right. \\
\Pi(X(i))=a^{-1}\left(G V\left(C L(i), X^{\prime}(i)\right) \oplus K L(i)\right) \\
V D\left(K R(i), X^{\prime}(i-1) \oplus X(i)\right)=a^{-1}\left(G V\left(C L(i), X^{\prime}(i)\right) \oplus K L(i)\right) \\
X^{\prime}(i-1) \oplus X(i)=D V\left(K R(i), a^{-1}\left(G V\left(C L(i), X^{\prime}(i)\right) \oplus K L(i)\right)\right) \\
X(i)=D V\left(K R(i), a^{-1}\left(G V\left(C L(i), X^{\prime}(i)\right) \oplus K L(i)\right)\right) \oplus X^{\prime}(i-1)
\end{array}\right.
$$

\section{AXIS6: EXAMPLES AND SIMULATIONS}

In order to measure the performance of our encryption system, we randomly select a large number of reference images, and then use our method to test them

\section{1) Brutal assaults}

They consist in reconstructing the encryption keys in a random manner.

\section{a) Key-space analysis}

The chaotic sequence used in our method ensures strong sensitivity to initial conditions and can protect it from any brutal attacks. The secret key to our system consists of

$$
\begin{array}{ll}
x_{0}=0,7655412001, & \mu_{1}=3.89231541 \\
y_{0}=0.865421331, & \mu_{2}=0,563215 \\
\mu_{3}=1,3561 & \mu_{4}=0,563215
\end{array}
$$

If we use single-precision real numbers $\mathbf{1 0}^{-\mathbf{1 0}}$ to operate, the total size of the key will greatly exceed $\approx \mathbf{2}^{\mathbf{1 8 0}} \gg \mathbf{2}^{\mathbf{1 1 0}}$, which is enough to avoid any brutal attacks.

\section{b) Secret key's sensitivity Analysis}

Our encryption key has a high sensitivity, which means that a small degradation of a single parameter used will automatically cause a large difference from the original image. The image below illustrates this confirmation 


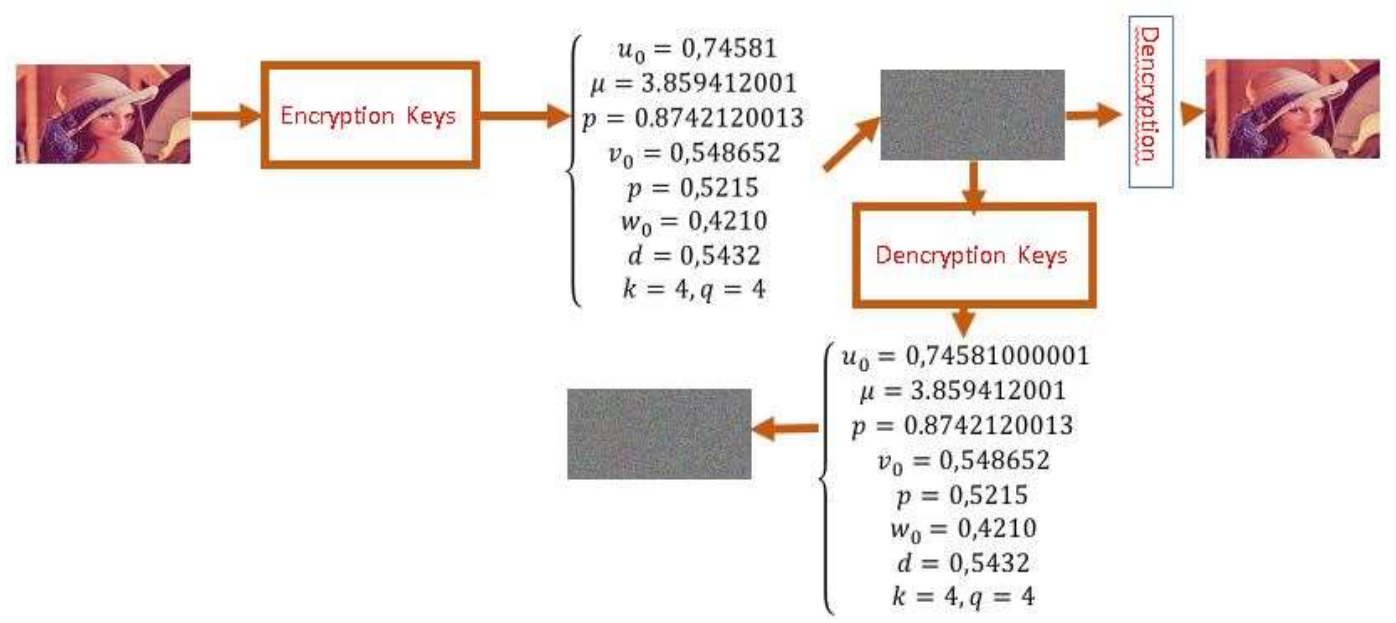

Figure 11: Encryption key sensitivity

This ensures that in the absence of the real encryption key, the original image cannot be restored.

\section{a) Hystogram analysis}

all images tested by our algorithm have a uniformly distributed histogram. This reflects that the entropy of the encrypted images is around 8, which makes the system immune to histogram attacks. The table 1 shows that the horizontal correlation values of the encrypted images are close to zero. This ensures high security against correlation attacks.

Table 1: Encrypted image histogram

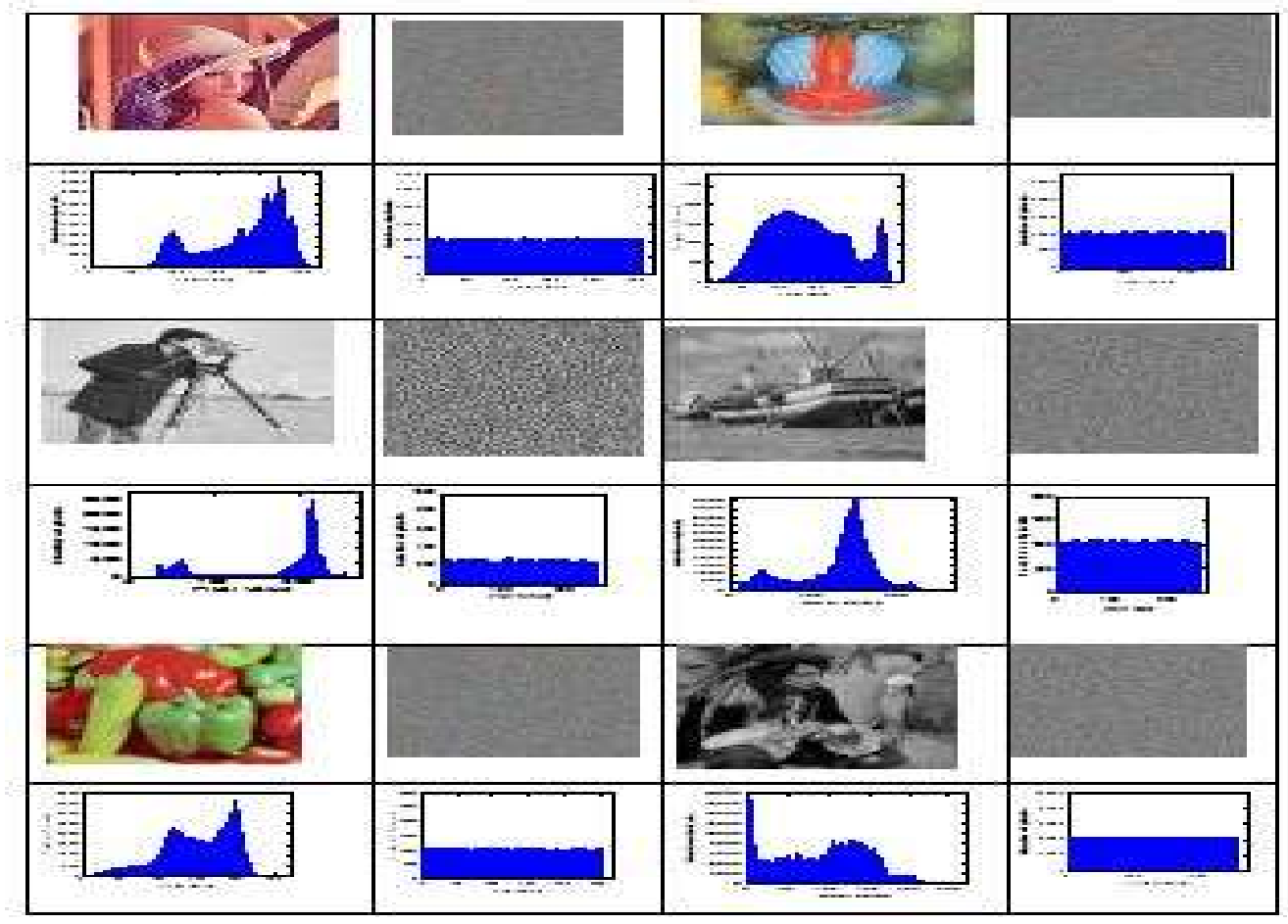

\section{2) Statistics Attack Security}

\section{a) Entropy Analysis}

Entropy is the measure of the disorder diffused by a source without memory. The entropy expression is determined by the equation below 
Equation 23

$$
H(M C)=\frac{1}{t} \sum_{i=1}^{t}-p(i) \log _{2}(p(i))
$$

The entropy values on the 150 images arbitrarily chosen from a large database of images of different sizes and formats, tested by our method are represented graphically by the following figure

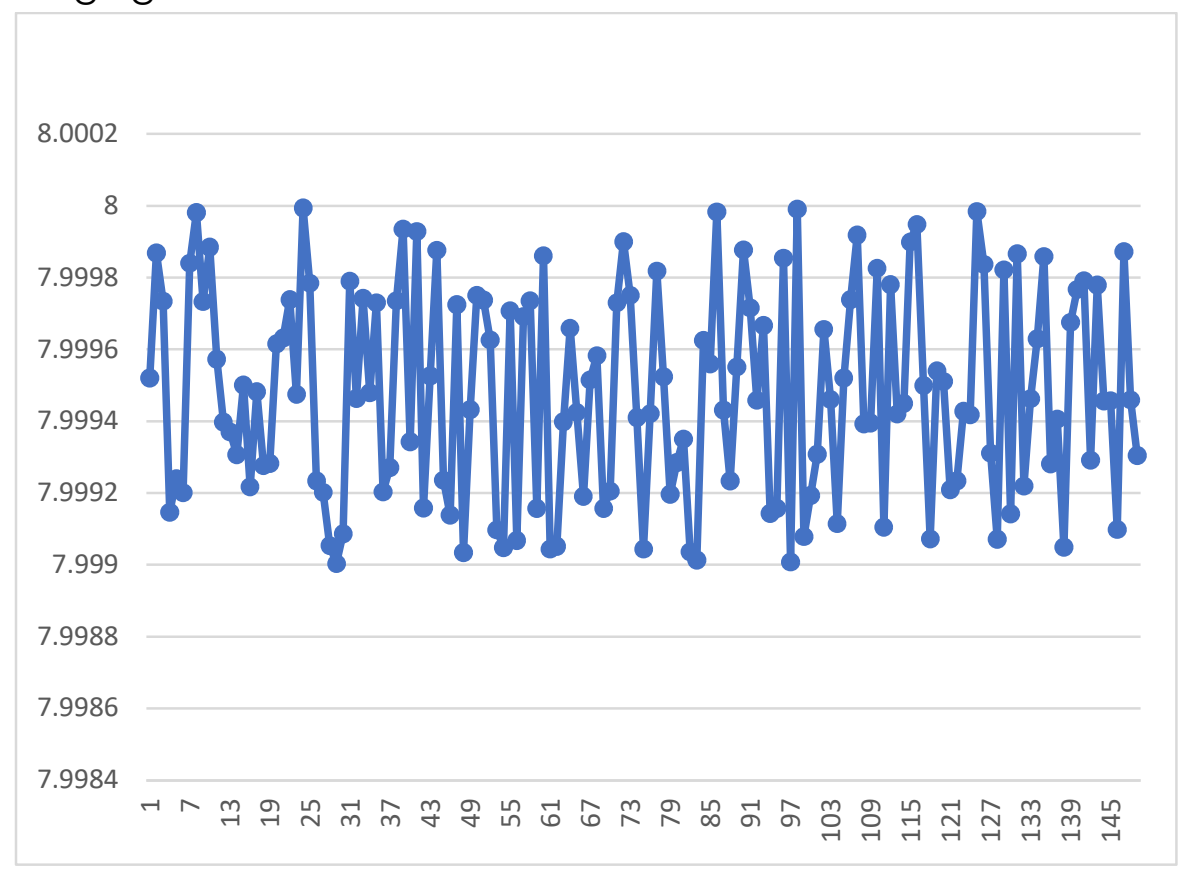

Fugure12: Entropy of 150 images

All the entropy values of the images tested by our algorithm are close to 8 , which confirms the uniformity of the histogram. This proves that the method is far from a statistical attack.

\section{a) Entropy statistical analysis}

We will study the uniformity of the distribution of entropy released by the test.

\section{(a) Position parameter analysis}

The values derived from the entropy by applying our approach to over 150 images in our image database, constitute a statistical series with position, dispersion and concentration parameters have been recalculated to verify the safety of our approach.

The purpose of this analysis is to show that the distribution follows a reduced central normal distribution. So

$$
\text { Equation } 24\left\{\begin{array}{l}
Q_{1}=\text { First quartile } \\
Q_{2}=\text { Second quartile } \\
Q_{3}=\text { Third quartile }
\end{array}\right.
$$




\section{Position Parameters}

\begin{tabular}{|c|c|c|c|c|c|}
\hline Average & Max & Min & Q1 & Q2 & Q3 \\
\hline 7,999480662 & 7,999993872 & 7,999004283 & 7,99921863 & 7,99946043 & 7,999737 \\
\hline
\end{tabular}

The moustache box of the entropy is illustrated in the diagram in Figure below

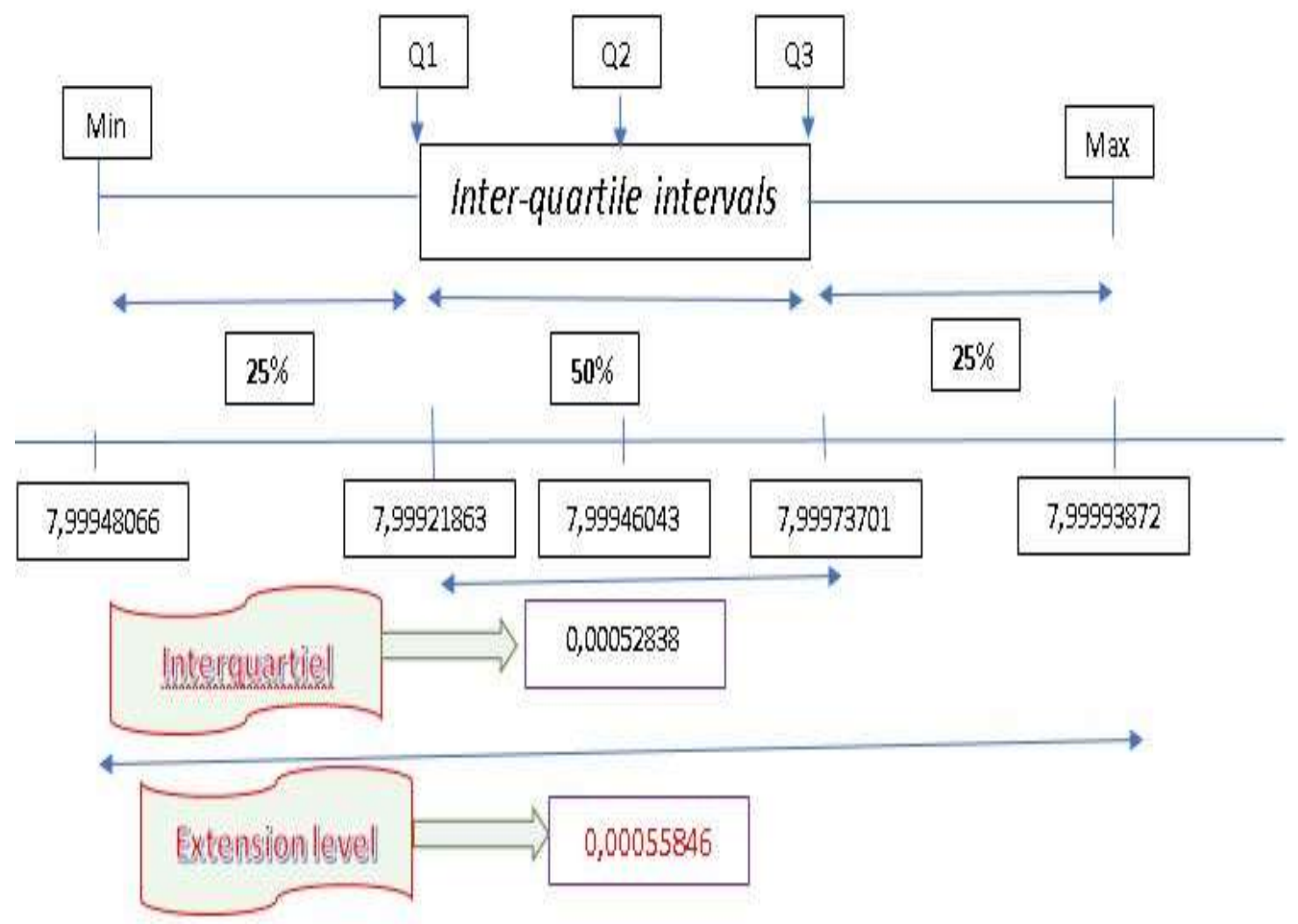

Figure13: Entropy moustache box

\section{a) Correlation analysis}

Correlation is a technique that compares two images to estimate the displacement of pixels in one image relative to another reference image. The relevant expression is defined by the following equation

$$
\text { equation } 29 \text { correlation } \quad r=\frac{\operatorname{cov}(x, y)}{\sqrt{V(x)} \sqrt{V(y)}}
$$

\section{(a) Horizontal Correlation}

The following figure graphically represents the simulation of 150 color images of the same size, which are selected from an image database of various sizes, formats and related values 


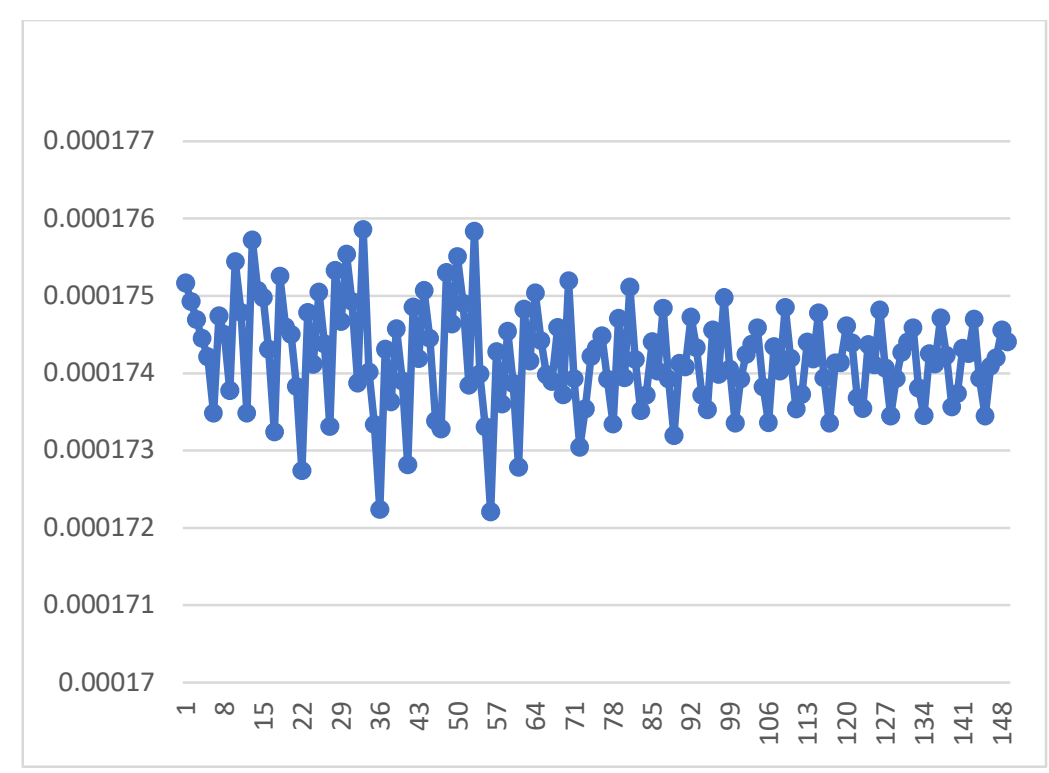

Fugure15: Entropy of 150 images

\section{(b) Vertical Correlation}

Simulations made on 150 images of the database gave the vertical correlation scores are displayed in Figure below

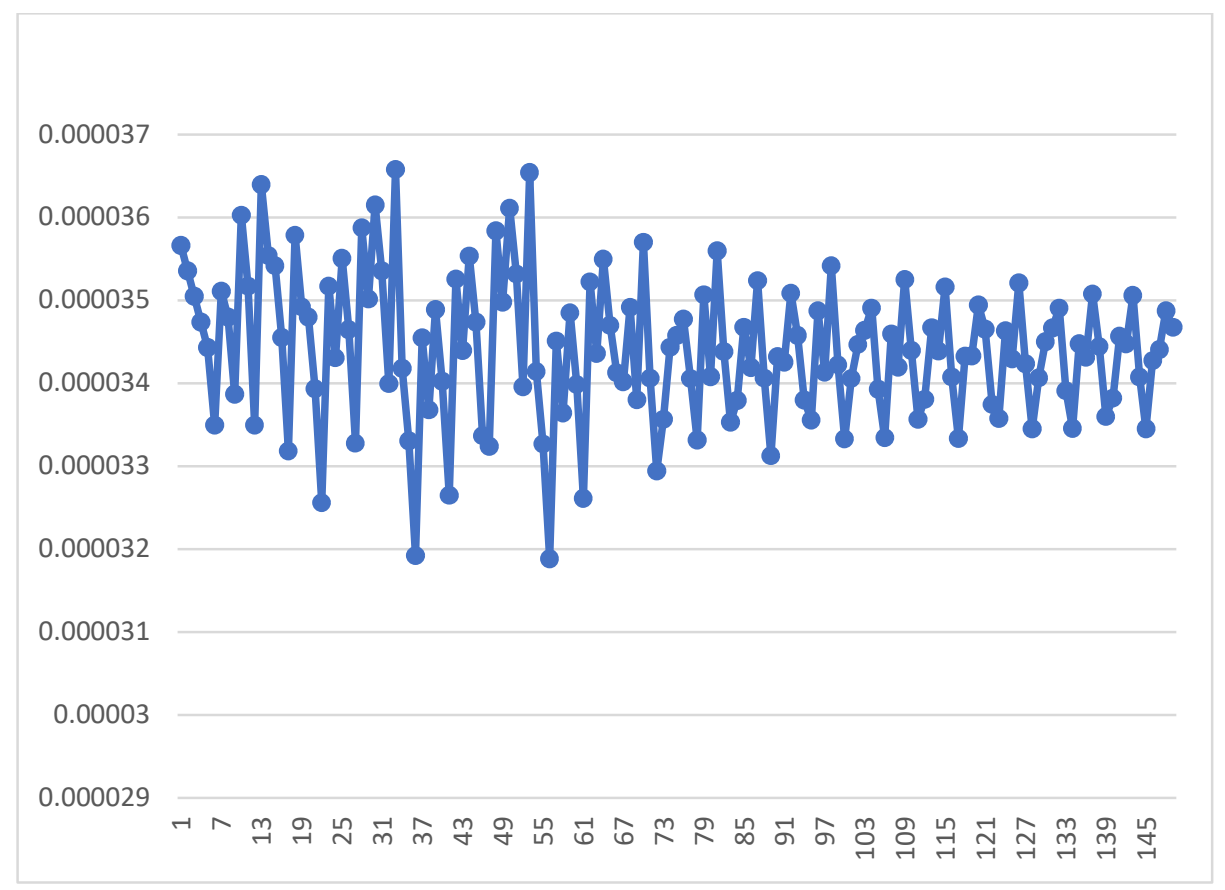

flgure16: Vertical correlation of 150 images

Figure 17 shows that the vertical correlation values of the encrypted images are close to zero. This ensures high security against correlation attacks.

\section{(c) Diagonal Correlation}

Simulations made on 150 images of the database gave the diagonal correlation scores are displayed in Figure below 


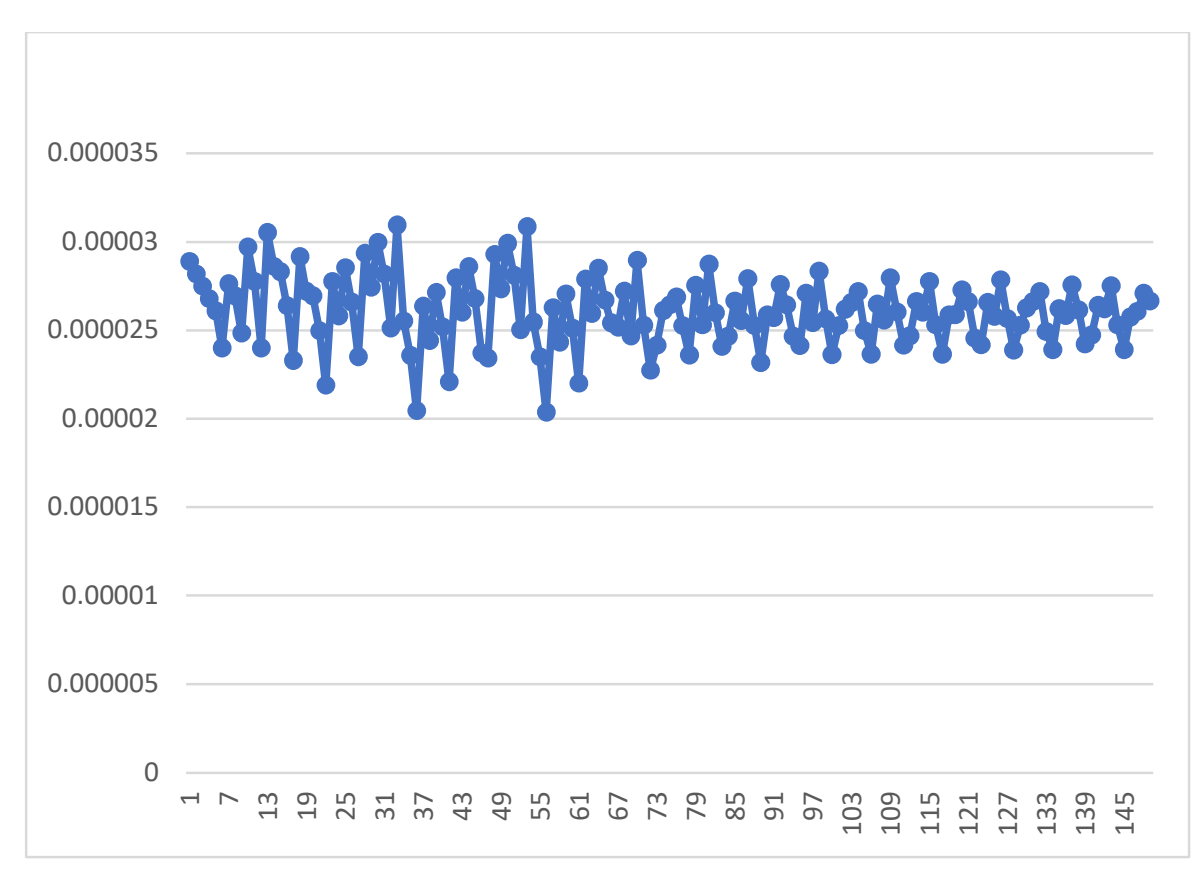

flgure17: Diagonal correlation of 150 images

Figure above shows that the diagonal correlation values of the encrypted images are close to zero. This ensures high security against correlation attacks.

\section{b) Differential analysis}

Let be two encrypted images, whose corresponding free-to-air images differ by only one pixel, from $\left(\boldsymbol{C}_{\boldsymbol{1}}\right)$ and $\left(\boldsymbol{C}_{\mathbf{2}}\right)$, respectively. The NPCR mathematical analysis of an image is given by the equation below

$$
\begin{array}{r}
\text { Equation } 30 \quad N P C R=\left(\frac{1}{n m} \sum_{i, j=1}^{n m} D(i, j)\right) * 100 \\
\text { With } D(i, j)=\left\{\begin{array}{lll}
1 & \text { if } & C_{1}(i, j) \neq C_{2}(i, j) \\
0 & \text { if } & C_{1}(i, j)=C_{2}(i, j)
\end{array}\right.
\end{array}
$$

The UACI mathematical analysis of an image is given by the below

$$
\text { Equation } 31 \quad U A C I=\left(\frac{1}{n m} \sum_{i, j=1}^{n m} \operatorname{Abs}\left(C_{1}(i, j)-C_{2}(i, j)\right)\right) * 100
$$

The study of the 150 selected images revealed the following diagram 


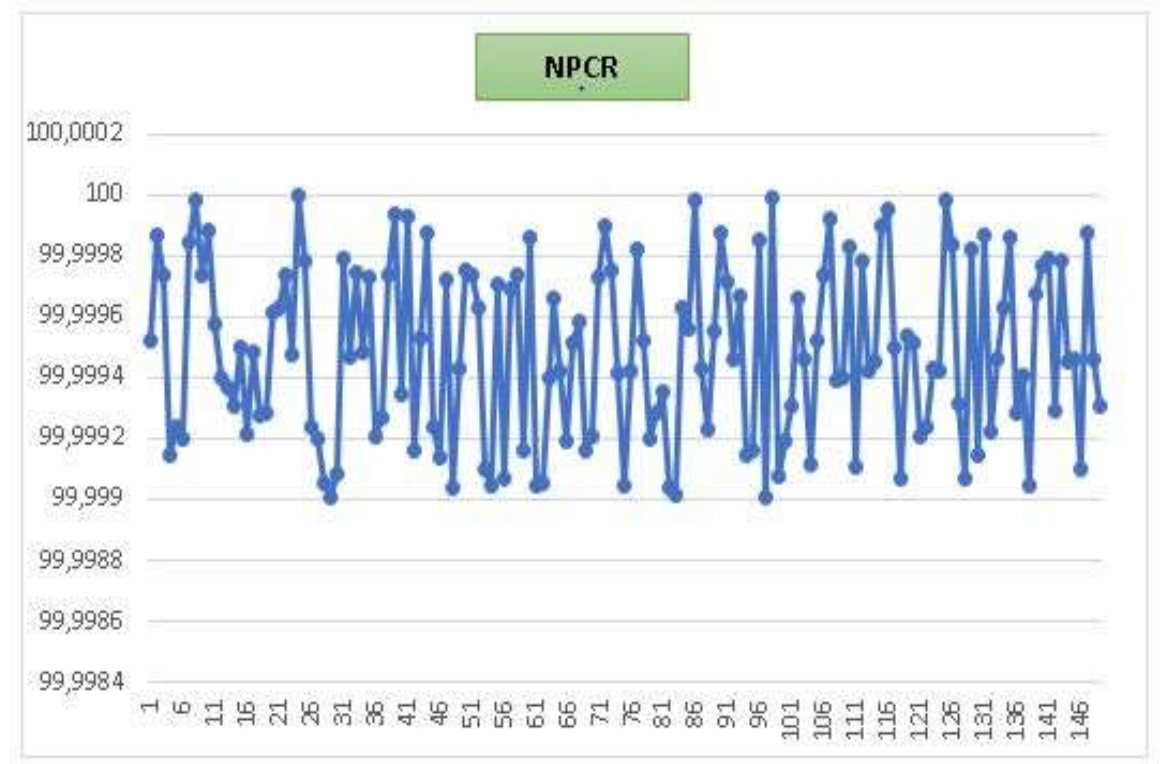

flgure18: NPCR of 150 images

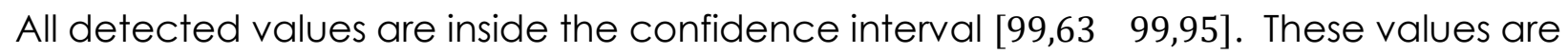
largely sufficient to affirm that our crypto system is protected from known differential attacks

The study of the 150 selected images revealed the following diagram

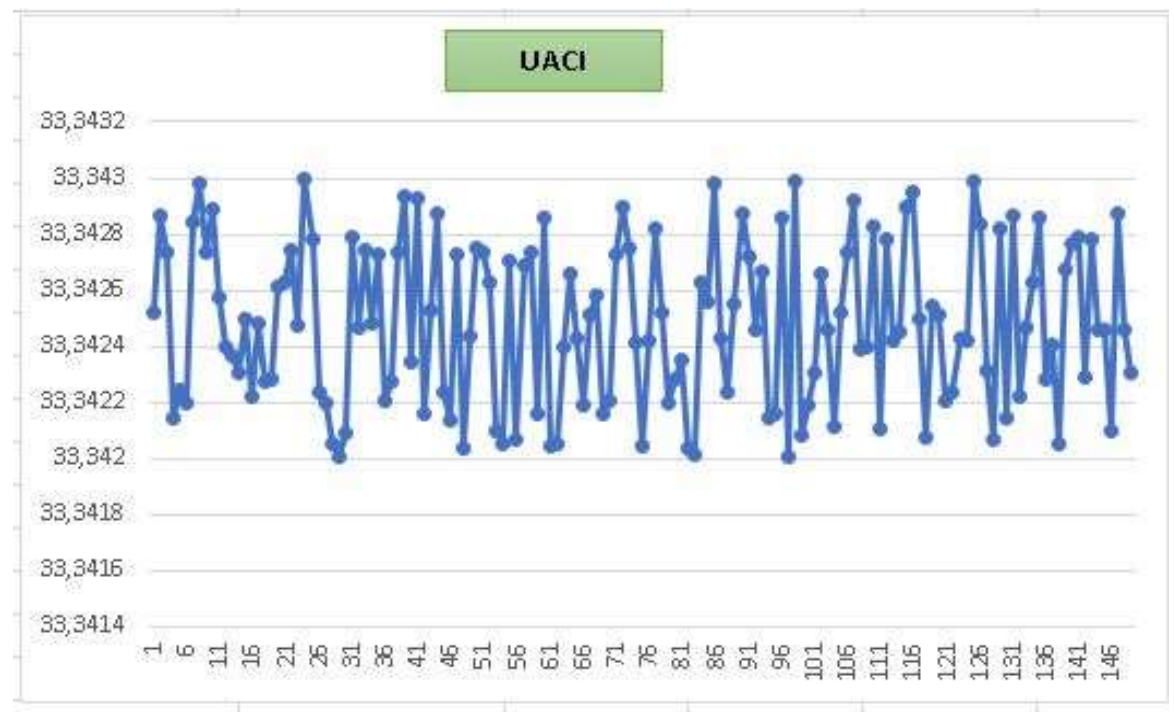

flgure19: UACl of 150 images

All detected values are inside the confidence interval [33;34 33,35]. These values are largely sufficient to affirm that our crypto system is protected from known differential attacks.

\section{a) Avalanche effect}

The avalanche effect is a required property in virtually all cryptographic hash functions and block coding algorithms. It causes progressively more important changes as the data is propagating in the structure of the algorithm. This constant determines the avalanche impact of the cryptographic structure in place. It is approximated by the equation below 


$$
\text { Equation32 } A E=\left(\frac{\sum_{i} \text { bit change }}{\sum_{i} \text { bit total }}\right) * 100
$$

Figure below depicts the evaluation of the $A E$ score for 150 images examined by our approach.

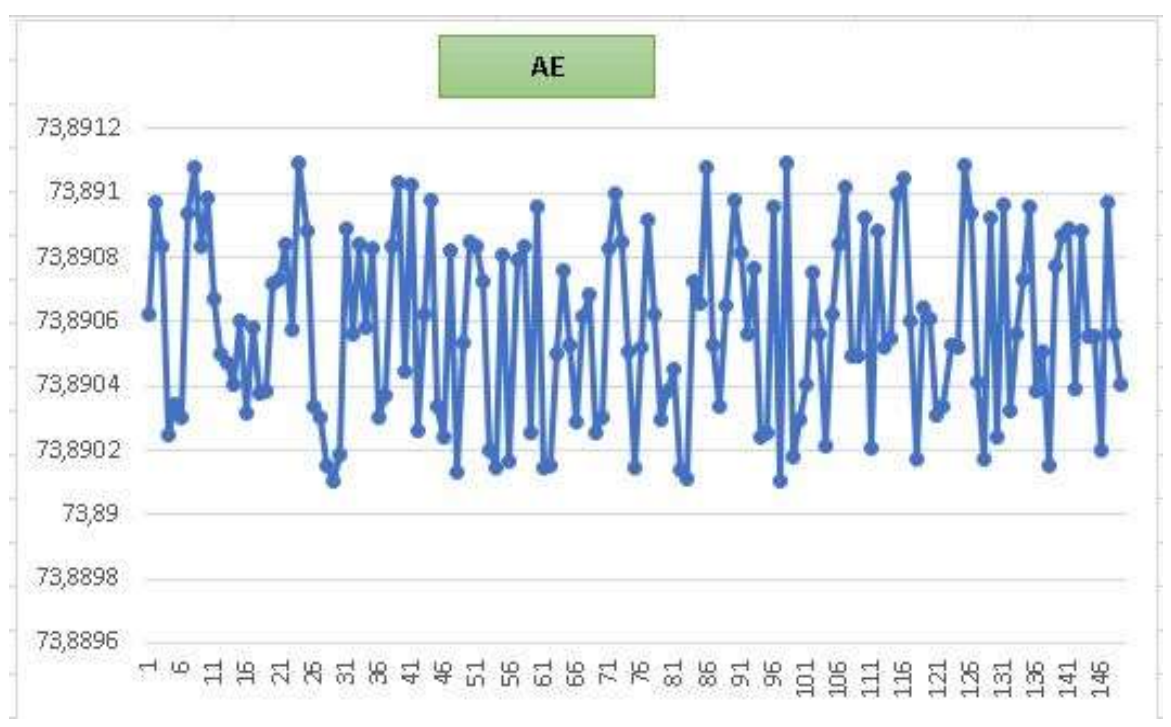

Figure20: Avalanche effect

All values returned from the $(A E)$ by our method are all in the range of residual values $[73,9674 ; 02]$. This guarantees that a one- bit change in the clear image will be reflected by a change of at least $78 \%$ of the encrypted image's bits.

\section{b) Signal-To-Peak Noise Ratio (PSNR)}

\section{(a) $M S E$}

The image quality estimation to be based on the pixel change was obtained by processing the (PSNR) values and the (MSE). It is calculated by the following equation

$$
\text { Equation } 33 M S E=\sum_{i, j}(P(i, j)-C(i, j))^{2}
$$

$(P(i, j))$; pixel of the clear image

$(C(i, j))$ : pixel of the cypher image

\section{(b) PSNR}

The signal-to-peak noise ratio, often abbreviated PSNR, is an engineering term for the ratio between a signal's maximum possible power and the power of distorted noise that affects the precision of its display. The PSNR mathematical analysis of an image is given by the next equation

$$
\text { Equation34 PSNR }=20 \log _{10}\left(\frac{I_{\max }}{\sqrt{M S E}}\right)
$$

For $R G B$ color images, the definition of $P S N R$ is the same except that the MSE is the sum of all square value changes. In the alternative, for color images, the image is transcoded 
into a separate color space and the PSNR is displayed for each channel in that color space.

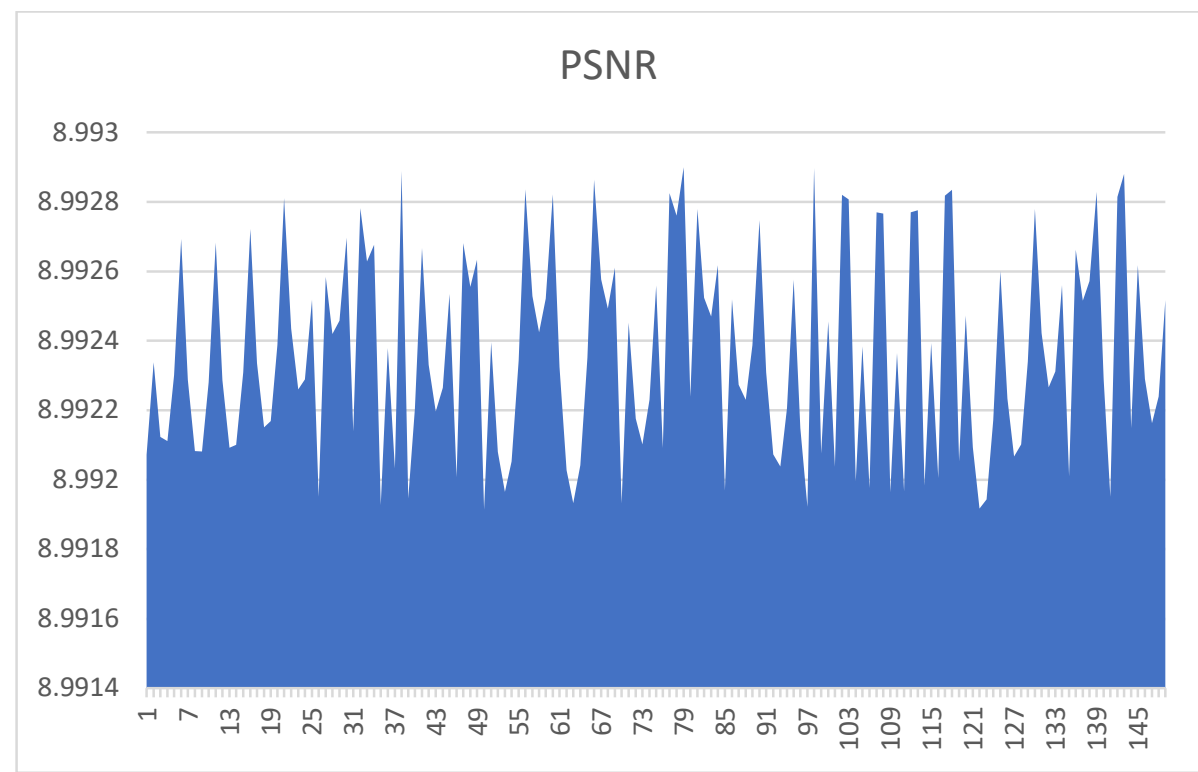

flgure21: "PSNR of 150 images

All values returned from the PSNR by our method are all in the range of residual values [8,99 8,993].

\section{a) Speed analysis}

For an evaluation of the execution time, our algorithm is tested on a personal computer "Intel core i5 3337 U 1.86 Gz CPU 8 GB ram. We use Matlab as programming software. We measure the encryption and decryption time of the tested images.

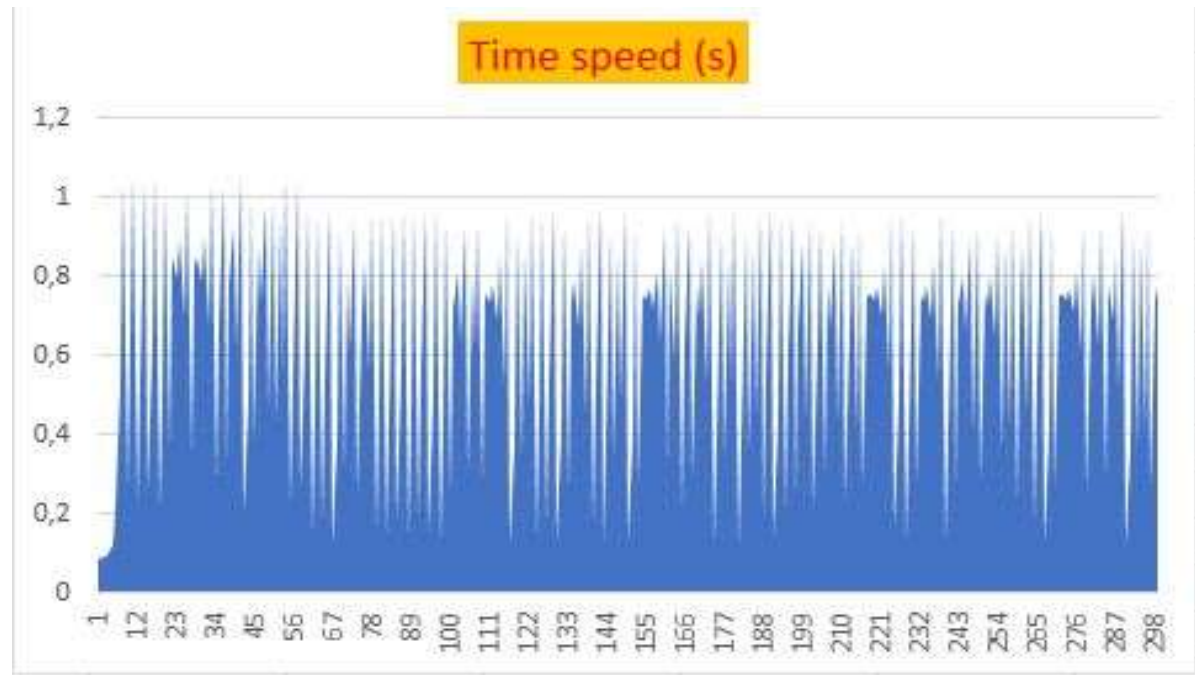

\section{4) Math Security}

\section{flgure22: time of 300 images}

The first round eliminates any correlations and protects the system from differential attacks. Not knowing the size of the encryption matrix will increase the complexity of the attack. Without knowing the encryption key, it is difficult to replicate the structure of this matrix. 


\section{CONCLUSION}

The first encryption stage is provided by the deep enhancement of Vigenere's classic system, which can protect our new algorithm from any differential attack, while the second stage uses dynamic affine transformation, which consists of a large invertible matrix of uncertain size Provide without knowing the secret encryption keys, are difficult to construct from Kronecker's products, making the system resistant to any known attacks. The system only uses the replacement, which greatly reduces the execution time.

\section{Conflict of Interest}

I am the sole author of this article, and there are no private or public organizations or laboratories to fund my research, thus avoiding any expected conflicts.

This document does not contain any research or experiments conducted on animals.

\section{References}

[1] A.P.USiahaan "Genetic algorithm in Hill cipher encryption"international association of scientific innovation and research (IASR) vol 15, no,1 2016

[2] A.S alkhalid " cryptanalyse of Hill cipher using genetic algorithm" dalam IEEE hanmument 2015 A.Jarjar « Improvement of hill' sclassical method in image cryptography » International Journal of Statistics and Applied Mathematics 2017, Volume 2 Issue 3, Part A Imam Saputra, Mesran, Nelly Astuti Hasibuan3, "Vigenere Cipher Algorithm with Grayscale Image Key

[4] Generator for Secure Text File" International Journal of Engineering Research \& Technology (IJERT), Vol. 6 Issue 01, January-2017 Vaka Vamshi Krishna Reddy, Sreedhar Bhukya 2, "ENCRYPT AND DECRYPT IMAGE USING VIGENERE CIPHER", International Journal of Pure and Applied Mathematics, Volume 118 No. 242018 A.P.U siahaan" three pass protocol in Hill cipher encryption technique" international journal of science and research (IJSR) vol 5 nà 72016 pp 1149-1152 I Gede Arya Putra Dewangga, Tito Waluyo Purboyo, Ratna Astuti Nugrahaeni," A New Approach of Data

[7] Hiding in BMP Image Using LSB Steganography and Caesar Vigenere Cipher Cryptography" International Journal of Applied Engineering Research, Volume 12, Number 21 (2017) pp. 10626-10636

[8] Overbey.J.traversW and W ydylo J 2005 "On the key space of the Hill cipher" Cryptologia 29(1), 59-72

[9]. Saeednia,S 2000 "haow to make the Hill secure" Cryptologia 24(2), 353-360

[10]. Lin.C.H.Lee.C.Y and Lee.C.Yu 2004" comments on saeednia's improved scheme for the Hill cipher." Journal of the chineese institute of engineers $27 / 5,743-746$ A.Jarjar « Improvement of hill's classical method in image cryptography » International Journal of Statistics and Applied Mathematics 2017, Volume 2 Issue 3, Part A IYongWang all " A chaos-based image encryption algorithm with variable control parameters" Chaos, Solitons \& Fractals Volume 41, Issue 4, 30 August 2009, Pages 1773-1783" Jan Sher Khan all, "Chaos based efficient selective image encryption" Multidimensional Systems and Signal Processing volume 30, pages943-961(2019)“

$\mathrm{H} \mathrm{Li}, \mathrm{Y}$ Wang, Z Zuo - Optics and Lasers in Engineering, 2019 "Chaos-based image encryption algorithm

[14] with orbit perturbation and dynamic state variable selection mechanisms" Volume 115, April 2019, Pages 197-207

[15] Rongjun Ge all « A Novel Chaos-Based Symme 
[16] Mohamed JarJar "Further improvement of the HILL method applied in image encryption" Procedia computer sciences 00(2019)000-000

Shams Mahmoud Abd Ali » Novel Encryption Algorithm for Securing Sensitive Information Based on

[17] Feistel Cipher"Test engeenering managenment Page Number: 10 - 16 Publication Issue:19 Volume: 80 September-October 2019

[18] Zhi-hua Gan » A chaotic image encryption algorithm based on 3-D bit-plane permutation » Neural Computing and Applications (2019) 31:7111-7130

[19] Khan JS, Ahmad J. Chaos based efficient selective image encryption. Multidimensional Systems and Signal Processing. 2019; 30(2): 943-961.

[20] Liu $\mathrm{H}$ « Color image encryption based on one time keys and robust chaotics maps" Computer and mathematic application 59(2010),3320-3327

[21] Liu H « Image encryption using DNA complementary rule and chaotic maps" Applied soft computing 12(2012)1457-1466

[22] Ingyuan Wang " Image encryption algorithm for synchronously updating Boolean network based on matrix semi tensor product" Information sciences 507/2020 16-35

[23] Ismail IA, Amin M, Diab H. A digital image encryption algorithm based a composition of two chaotic logistic maps. IJ Network Security. 2010; 11(1): 1-10. 